Canadian Science Publishing

Biochemistry and Cell Biology

Biochimie et biologie cellulaire

\title{
Ceruloplasmin-derived peptide is the strongest regulator of oxidative stress and leukotriene synthesis in neutrophils
}

\begin{tabular}{|r|l|}
\hline Journal: & Biochemistry and Cell Biology \\
\hline Manuscript ID & bcb-2016-0180.R1 \\
\hline Manuscript Type: & Note \\
\hline Domplete List of Authors: & $\begin{array}{l}\text { Golenkina, Ekaterina; Lomonosov Moscow State University, Belozersky } \\
\text { Institute of Physico-Chemical Biology } \\
\text { Livenskyi, Alexey; Lomonosov Moscow State University, Belozersky } \\
\text { Institute of Physico-Chemical Biology } \\
\text { Viryasova, Galina; Lomonosov Moscow State University, Belozersky } \\
\text { Institute of Physico-Chemical Biology } \\
\text { Romanova, Yulia; Gamaleya Research Institute of Epidemiology and } \\
\text { Microbiology } \\
\text { Sud'ina, Galina; Lomonosov Moscow State University, Belozersky Institute } \\
\text { of Physico-Chemical Biology } \\
\text { Sokolov, Alexey; Institute for Experimental Medicine, Molecular Genetic; } \\
\text { Saint-Petersburg State University }\end{array}$ \\
\hline Keyword: & \begin{tabular}{l} 
Ceruloplasmin, 5-lipoxygenase, myeloperoxidase, superoxide, neutrophil \\
\hline
\end{tabular} \\
\hline
\end{tabular}

\section{SCHOLARONE ${ }^{m}$ \\ Manuscripts}


1 Ceruloplasmin-derived peptide is the strongest regulator of oxidative stress and leukotriene

2 synthesis in neutrophils

3 Ekaterina A. Golenkina ${ }^{\mathrm{a}}$, Alexey D. Livenskyi ${ }^{\mathrm{a}}$, Galina M. Viryasova ${ }^{\mathrm{a}}$, Yulia M. Romanova ${ }^{\mathrm{b}}$,

4 Galina F. Sud'ina ${ }^{a} *$, Alexey V. Sokolov ${ }^{\mathrm{c}, \mathrm{d}}$

5

6 a Lomonosov Moscow State University, Belozersky Institute of Physico-Chemical Biology,

7 Moscow 119234, Russia

$8 \quad{ }^{\mathrm{b}}$ Gamaleya Research Institute of Epidemiology and Microbiology, Moscow 123098, Russia

$9 \quad{ }^{\text {c} F S B S I ~ “ I n s t i t u t e ~ o f ~ E x p e r i m e n t a l ~ M e d i c i n e ”, ~ S t . ~ P e t e r s b u r g ~ 197376, ~ R u s s i a ~}$

10 dSaint-Petersburg State University, St. Petersburg 199034, Russia

11

12 Corresponding author: Galina F. Sud'ina*

13 *Lomonosov Moscow State University, Belozersky Institute of Physico-Chemical Biology,

14 Moscow 119234, Russia; Tel +7-495-939-5359; Fax +7-495-939-3181; E-mail:

15

16

17 


\section{Abstract}

19 Ceruloplasmin, an acute-phase protein, can affect the activity of leukocytes through its various

enzymatic activities and protein-protein interactions (with lactoferrin, myeloperoxidase,

21 eosinophil peroxidase, serprocidins, and 5-lipoxygenase (5-LOX), among others). However, the

22 molecular mechanisms of ceruloplasmin activity are not clearly understood. In this study, we

23 tested the ability of two synthetic peptides, RPYLKVFNPR (883-892) (P1) and

RRPYLKVFNPRR (882-893) (P2), corresponding to the indicated fragments of the

ceruloplasmin sequence, to affect neutrophil activation. Leukotriene (LT) B4 is the primary

eicosanoid product of polymorphonuclear leukocytes (PMNLs, neutrophils).We studied

leukotriene synthesis in PMNLs upon interaction with Salmonella enterica serovar

Typhimurium. Priming of neutrophils with phorbol 12-myristate 13-acetate (PMA) elicited the

strong regulatory function of $\mathrm{P} 2$ peptide as a superoxide formation inducer and leukotriene synthesis inhibitor. Ceruloplasmin-derived P2 peptide appeared to be a strong inhibitor of 5LOX product synthesis under conditions of oxidative stress.

\section{Keywords}

33 Ceruloplasmin; 5-lipoxygenase; myeloperoxidase; superoxide; neutrophil

\section{Abbreviations}

PMNL, polymorphonuclear leukocyte; Cp, ceruloplasmin; MPO, myeloperoxidase; LTB4, leukotriene B4; 5-LOX, 5-lipoxygenase; PMA, phorbol 12-myristate 13-acetate; ROS, reactive lipopolysaccharide; NO, nitric oxide 
41

42

\section{Introduction}

Ceruloplasmin $(\mathrm{Cp})$ is an acute-phase plasma protein with both antioxidant and prooxidant activities (Samygina VR et al. 2013). Cp can participate in protein-protein interactions with a number of leukocyte and pro-inflammatory proteins, including myeloperoxidase (MPO), lactoferrin serprocidins, eosinophilic cationic protein and 5-lipoxygenase (5-LOX) (Sokolov et al. 2007; Sokolov et al. 2010). Complexes of Cp with lactoferrin, MPO, and apoB100-containing lipoproteins were detected in the plasma of patients with inflammatory diseases and atherosclerosis (Sokolov et al. 2014). At the blood level, Cp plays a number of protective and antioxidant roles (Adamsson et al. 2011). For example, $\mathrm{Cp}$ reduces the generation of reactive oxygen species (ROS) and nitrogen species (Shiva et al. 2006). Moreover, Cp maintains plasma nitrite (which acts as a substrate pool for nitric oxide (Varfolomeeva et al. 2016) formation) by catalyzing the oxidation of $\mathrm{NO}$ to $\mathrm{NO}+$ and subsequent hydration to nitrite (Shiva et al. 2006).

Endogenous $\mathrm{Cp}$, which usually plays an anti-inflammatory/antioxidant role, if present at increased concentrations, could exacerbate the damaging effects of pro-inflammatory stimuli in the brain by increasing lipopolysaccharide (LPS)-induced NO production in microglial cells (Lazzaro et al. 2014). Finally, $\mathrm{Cp}$ acts as a superoxide dismutase (SOD) to affect the production of ROS by neutrophils (Sokolov et al. 2010).

ROS, such as superoxide and hydrogen peroxide, are generated by free iron (via Fenton and Haber Weiss-type reactions) and inducible enzymes, such as xanthine and NADPH oxidases (Segal 2005). MPO is known to be activated by hydrogen peroxide (Flemmig and Arnhold 2010). MPO undergoes numerous reactions, including halogenation, peroxidase, catalase, and SOD reactions (Arnhold and Flemmig 2010). Ferric $\left(\mathrm{Fe}^{3+}\right)$ MPO reacts with superoxide anion radicals and is able to function as a SOD (Winterbourn et al. 1985; Kettle et al. 2007). Among plasma proteins, $\mathrm{Cp}$ shows the strongest effects on inhibition of MPO activity (Govorova et al. 1989). 

accumulation of NADPH oxidase on cell surfaces (Segal 2005). A powerful trigger of NADPH

oxidase in leukocytes is phorbol 12-myristate 13-acetate (PMA). Upon phagocytosis, the activation of NADPH oxidase proceeds in the walls of the phagocytic vacuole, where it forms a conduit for electrons to be pumped from NADPH in the cytosol onto oxygen in the vacuole (Segal 2005), facilitating the release of protease enzymes from cytoplasmic granules. Initiation of NADPH oxidase activity coincides with degranulation, initially for azurophilic (or primary) granules that largely contain MPO and proteins directed toward microbial killing and digestion (e.g., cathepsin G, elastase, and proteinase 3).

Following the onset of infection, PMNLs mount the first antimicrobial attack via phagocytosis. 5- LOX is activated during the phagocytosis of microorganisms and foreign particles by neutrophils (Williams et al. 1985). Upon cell activation, human neutrophils readily produce leukotriene (LT) B4. LTs are lipid mediators derived from arachidonic acid via the 5LOX pathway. In a previous study, we investigated the interaction of $\mathrm{Cp}$ with 5-LOX and found that Cp inhibits 5-LOX (Sokolov et al. 2010).

$\mathrm{Cp}$ has been shown to act as a physiological inhibitor of myeloperoxidase (MPO) (Segelmark et al. 1997). The heme protein MPO is highly specific to neutrophils (Klebanoff 2005). MPO is abundantly present in azurophil granules of neutrophils that fuse with phagocytosed bacteria. Moreover, the formation of a specific complex between Cp and MPO has been described (Samygina et al. 2013). In the 2Cp-MPO complex, the MPO heme pocket is shielded by a fragment of Cp (sequence 883-892) (Samygina et al. 2013). A synthetic peptide (RPYLKVFNPR), corresponding to the fragment of $\mathrm{Cp}$ at positions $883-892$, is a highly efficient inhibitor of MPO and inhibits the peroxidase and chlorinating activities of MPO with half-maximal inhibitory concentrations ( $\mathrm{IC}_{50}$ values) of 160 and $780 \mathrm{nM}$, respectively (Samygina et al. 2013; Sokolov et al. 2015b). MPO-derived oxidants have dramatic effects on the product 
91 profiles of human 5-LOX (Zschaler et al. 2015); therefore, targeting of MPO with specific

92 peptides may regulate leukotriene synthesis.

93 In this study, we tested the ability of two synthetic peptides, RPYLKVFNPR (883-892)

94 and RRPYLKVFNPRR (882-893), to modulate superoxide production and leukotriene synthesis

95 in neutrophils.

96

97

\section{Materials and Methods}

98

99

100

101

102

103

104

105

106

107

108

109

110

111

112

\subsection{Bacteria and reagents}

Salmonella Typhimurium strain IE 147 was obtained from the Collection of Gamaleya Research Centre of Epidemiology and Microbiology (Moscow, Russia). Bacteria were grown in Luria-Bertani broth, washed twice using physiological salt solution, and centrifuged at $2000 \times g$. The concentration of the stock suspension was $1 \times 10^{9} \mathrm{CFU} / \mathrm{mL}$. The bacteria were opsonized with fresh serum from the same donor whose blood was used to prepare neutrophils. Serum was isolated by centrifuging clotted blood. For opsonization, bacteria were incubated for $30 \mathrm{~min}$ in Dulbecco's salt solution containing 5\% serum. Hank's balanced salt solution with calcium and magnesium but without Phenol Red and sodium hydrogen carbonate (HBSS), Dulbecco's PBS with magnesium but without calcium, PMA, and cytochrome c (Cyto C) were purchased from Sigma (Steinheim, Germany). Dextran T-500 was from Pharmacosmos (Holbæk, Denmark). In this study, we used synthetic amino acid sequences purified by high-performance liquid chromatography (HPLC; NPF Verta, Russia).

The amino acid sequences of the peptides used in this study are presented in Table 1.

Table 1. Amino acid sequences of the peptides used in this study.

\begin{tabular}{|lll|}
\hline Abbreviation & Amino acid sequence & Molecular mass (Da) \\
P1 & RPYLKVFNPR (883-892) & 1290 \\
\hline
\end{tabular}




P2 RRPYKVFNPRR (882-893) 1600

\subsection{Ethics statement}

We prepared neutrophils from the blood of healthy volunteers. Blood was collected via venous puncture, as approved by the Ministry of Public Health Service of the Russian Federation. Experimental and participant consent procedures were approved by the Institutional Ethics Committee of the A. N. Belozersky Institute of Physico-Chemical Biology, Moscow State University.

\subsection{Human neutrophil isolation}

We isolated PMNLs from freshly drawn citrate-anticoagulated donor blood using standard techniques (Sud'ina et al. 2001). We prepared leukocyte-rich plasma by sedimentation of red blood cells with 3\% dextran T-500 at room temperature. We purified granulocytes by centrifugation of leukocyte-rich plasma through Ficoll-Paque (density: $1.077 \mathrm{~g} / \mathrm{mL}$ ) followed by hypotonic lysis of the remaining red blood cells (RBCs). PMNLs were washed twice with phosphate-buffered saline (PBS), resuspended at $10^{7} / \mathrm{mL}$ (purity: 96-97\%, viability: 98-99\%) in Dulbecco's PBS containing $1 \mathrm{mg} / \mathrm{mL}$ glucose (without $\mathrm{CaCl}_{2}$ ), and stored at room temperature.

\subsection{Analysis of arachidonic acid metabolism via the 5-LOX pathway}

PMNLs $\left(2 \times 10^{7}\right.$ cells $)$ were incubated in $6 \mathrm{~mL}$ HBSS/HEPES medium at $37^{\circ} \mathrm{C}$ with or without tested compounds for $30 \mathrm{~min}$. Thereafter, Salmonella Typhimurium was added at 2550:1 (bacteria:PMNLs) for 20 min. Incubation was terminated by addition of an equal volume of methanol at $-20^{\circ} \mathrm{C}$. Prostaglandin $\mathrm{B}_{2}$ served as an internal standard. The samples were stored at $20^{\circ} \mathrm{C}$. The denatured cell suspensions were centrifuged, and the supernatants were collected as the water/methanol extracts. 

columns (500 mg; Macherey-Nagel, Dueren, Germany), which were conditioned sequentially with methanol and water. Metabolites of 5-LOX were extracted with $1.5 \mathrm{~mL}$ methanol. The samples were evaporated, dissolved in $35 \mu \mathrm{L}$ methanol/water (2:1), and analysed by reversedphase HPLC, as described previously (Viryasova et al. 2014).

\subsection{Measurement of superoxide anion production by Cyto c reduction}

PMNL incubations were performed in collagen-coated 24-well culture plates. The

HBSS/HEPES medium was supplemented with $50 \mu \mathrm{M}$ Cyto c, test compounds, $50 \mathrm{U} / \mathrm{mL}$ SOD,

$\mathrm{CO}_{2}$ incubator. The reactions were then stopped by cooling to $4^{\circ} \mathrm{C}$, and Cyto c reduction was measured as the increase in $\Delta 555 / 531$ (the absorbance at 555 minus the absorbance at $531 \mathrm{~nm}$ ).

Reduction of $10 \mu \mathrm{M}$ Cyto c caused an increase in the $\Delta 550 / 535$ by 0.18 absorbance units.

\subsection{Measurement of ROS}

Intracellular formation of ROS was monitored by fluorometric measurements of green

\subsection{Statistical analysis} significant.

\section{Results and Discussion}


160

161

162

163

164

165

166

167

168

173

174

175

176

177

\subsection{Priming of PMNLs with PMA elicited the effects of P2 on 5-LOX product formation upon} treatment with Salmonella Typhimurium

Addition of P1 and P2 to PMNLs did not induce 5-LOX product formation in neutrophils (data not shown). During phagocytosis of bacteria such as Salmonella Typhimurium, human neutrophils synthesize 5-LOX metabolites (LTB4 and its isomers, $\omega$-OH-LTB4 and 5-HETE) (Golenkina et al. 2011). PMNL incubation with opsonised Salmonella Typhimurium at a ratio of bacteria:PMNLs in the range 25-50:1 resulted in the production of 5-LOX metabolites; the data are presented in Fig. 1. Pretreatment of PMNLs with P1 or P2 did not influence the synthesis of 5-LOX products in PMNLs exposed to bacteria, except for increased LTB4 synthesis after treatment with P1 (Fig. 1). Pre-incubation (priming) of cells with 0.5 nM PMA augmented the synthesis of 5-LOX products. At concentrations below $1 \mathrm{nM}$, PMA is essentially nontoxic for neutrophils (Takei et al. 1996). In the presence of PMA, P2 appeared to exert strong suppressive effects on LT synthesis (Fig. 1).

\subsection{P2 activated superoxide formation in neutrophils upon their interaction with PMA}

PMA stimulates the production of $\mathrm{O}_{2}^{-}$by human neutrophils with $\mathrm{ED}_{50}$ concentrations of $3.9 \pm 2.1 \mathrm{nM}$ (Tauber et al. 1982). P1 and P2 did not induce superoxide formation in neutrophils, as measured by SOD-dependent Cyto c reduction. We stimulated superoxide formation by adding $0.5 \mathrm{nM}$ PMA to PMNLs. In the presence of $20 \mu \mathrm{M}$ P2, we observed a significant (more than two-fold) increase in PMA-induced superoxide formation (Fig. 2). Application of the superoxide-scavenger SOD $(30 \mathrm{U} / \mathrm{mL})$ to the cells completely blocked Cyto c reduction in all samples (data presented for PMA only).

Using carboxy-H2DCFDA as an indicator of oxidative stress in the cell, we observed increased ROS formation in PMNLs stimulated with PMA in the presence of the peptides (Fig. 3). Without PMA, P1 and P2 decreased the level of ROS in PMNLs (Fig. 3). 

Treatment with the peptides and PMA yielded a sharp jump in superoxide release and ROS formation, possibly through the activity of the peptide on the antioxidant systems of neutrophils. Antioxidant systems include SOD, catalase, and glutathione (Lubrano and Balzan 2015). P1 is a highly specific inhibitor of MPO (Samygina et al. 2013), which is abundantly expressed in neutrophils, and the observed slight decrease in ROS (Fig. 3) may be connected with this inhibitory activity. When neutrophils ingest bacteria or are exposed to PMA, they

able to function as an SOD (Winterbourn et al. 1985; Kettle et al. 2007). It is possible that inhibition of this activity causes oxidative stress in the cell when the elimination of $\mathrm{O}_{2}{ }^{-}$and $\mathrm{H}_{2} \mathrm{O}_{2}$ is blocked (Scheme 1). P2 peptide, which has two arginines at each end (RRPYLKVFNPRR 882-893), exhibited even stronger activation of PMA-induced oxidative stress.

The effects of $\mathrm{P} 1$ and $\mathrm{P} 2$ on ROS production differ from the effects of the intact $\mathrm{Cp}$ protein. The latter can act as an SOD and inhibit the activities of MPO and 5-LOX, thereby decreasing the generation of ROS and $\mathrm{HOCl}$ in PMNLs. As we showed in previous studies, limited proteolysis of Cp affects peptide bonds, particularly between K887 and V888, and prevents inhibition of MPO and 5-LOX (Sokolov et al. 2010; Sokolov et al. 2015a). In this study, we demonstrated that inhibition of MPO by P1 and P2 in the context of PMA-induced oxidative stress resulted in increased ROS production and decreased LT synthesis.

The activity of 5-LOX and synthesis of LTs are sensitive to ROS and the 'peroxide tone' of the cell (Weitzel and Wendel 1993), including MPO-derived oxidants (Zschaler et al. 2015). In our current study, we analyzed the synthesis of LTs by human PMNLs during their interaction with Salmonella Typhimurium. As part of the innate immune system, PMNLs kill Salmonella in a complement-dependent manner. Priming of PMNLs with PMA enhances LT synthesis induced by bacteria or zymosan particles (Viryasova et al. 2014; Viryasova et al. 2016). In cells challenged with the calcium ionophore A23187, 5-LOX product formation is inhibited by PMA and is not dependent on addition of the MPO inhibitor ABAH (Zschaler and Arnhold 2016). Cp- 
211 derived P2 peptide appeared to be a strong inhibitor of 5-LOX product synthesis during the

212 interaction with bacteria Salmonella Typhimurium under conditions of oxidative stress.

213 In summary, our results showed that low-molecular-weight derivatives of Cp exhibited

214 high activity in the regulation of neutrophil cellular responses. We treated PMNLs with two

215 ceruloplasmin-derived peptides, RPYLKVFNPR (883-892) (P1) and RRPYLKVFNPRR (882-

216 893) (P2) and found that neutrophil priming with PMA revealed the regulatory roles of the

217 peptides. P1 and P2 increased PMA-induced ROS formation in PMNLs. ROS are primarily

218 produced via NADPH oxidase and play an important role in the killing of microorganisms by

219 neutrophils. Peptide P2 inhibited 5-LOX product synthesis under conditions of oxidative stress.

220 In the inflammatory loci, where excessive activation of neutrophils results in oxidative stress in

221 cells, Cp P2 peptide would be expected to suppress LT synthesis. Thus, because LTB4 is a

222 chemoattractant for neutrophils themselves, treatment with P2 would block neutrophil

223 accumulation.

\section{Acknowledgements}

This study was funded by grant 13-04-00496 from the Russian Foundation for Basic Research.

\section{References}

Adamsson, S., Smith, J.G., Melander, O., Hedblad, B., and Engstrom, G. 2011. Inflammation-sensitive proteins and risk of atrial fibrillation: a population-based cohort study. Eur J Epidemiol 26: 449455. doi: 10.1007/s10654-011-9565-6.

Arnhold, J., and Flemmig, J. 2010. Human myeloperoxidase in innate and acquired immunity. Arch Biochem Biophys 500: 92-106. doi: 10.1016/j.abb.2010.04.008.

Flemmig, J., and Arnhold, J. 2010. Interaction of hypochlorous acid and myeloperoxidase with phosphatidylserine in the presence of ammonium ions. J Inorg Biochem 104: 759-764. doi: 10.1016/j.jinorgbio.2010.03.009.

Golenkina, E.A., Galkina, S.I., Romanova, J.M., Lazarenko, M.I., and Sud'ina, G.F. 2011. Involvement of red blood cells in the regulation of leukotriene synthesis in polymorphonuclear leucocytes upon interaction with Salmonella Typhimurium. APMIS 119: 635-642. doi: 10.1111/j.16000463.2011.02786.x. 
Govorova, N.Iu., Sharonov, B.P., and Lyzlova, S.N. 1989. Erythrocyte oxidative damage by myeloperoxidase. The protective action of serum proteins. Biull Eksp Biol Med 107: 428-430. doi: 10.1007/BF00842381.

Kettle, A.J., Anderson, R.F., Hampton, M.B., and Winterbourn, C.C. 2007. Reactions of superoxide with myeloperoxidase. Biochemistry 46: 4888-4897. doi: 10.1021/bi602587k.

Klebanoff, S.J. 2005. Myeloperoxidase: friend and foe. J Leukoc Biol 77: 598-625. doi: 10.1189/jlb.1204697.

Lazzaro, M., Bettegazzi, B., Barbariga, M., Codazzi, F., Zacchetti, D., and Alessio, M. 2014. Ceruloplasmin potentiates nitric oxide synthase activity and cytokine secretion in activated microglia. J Neuroinflammation 11: 164. doi: 10.1186/s12974-014-0164-9.

Lubrano, V., and Balzan, S. 2015. Enzymatic antioxidant system in vascular inflammation and coronary artery disease. World J Exp Med 5: 218-224. doi: 10.5493/wjem.v5.i4.218.

Samygina, V.R., Sokolov, A.V., Bourenkov, G., Petoukhov, M.V., Pulina, M.O., Zakharova, E.T., Vasilyev, V.B., Bartunik, H., and Svergun D.I. 2013. Ceruloplasmin: macromolecular assemblies with ironcontaining acute phase proteins. PLoS One 8: e67145. doi: 10.1371/journal.pone.0067145.

Segal, A.W. 2005. How neutrophils kill microbes. Annu Rev Immunol 23: 197-223. doi: 10.1146/annurev.immunol.23.021704.115653.

Segelmark, M., Persson, B., Hellmark, T., and Wieslander, J. 1997. Binding and inhibition of myeloperoxidase (MPO): a major function of ceruloplasmin? Clin Exp Immunol 108: 167-174. doi: 10.1046/j.1365-2249.1997.d01-992.x.

Shiva, S., Wang, X., Ringwood, L.A., Xu, X., Yuditskaya, S., Annavajjhala, V., Miyajima, H., Hogg, N., Harris, Z.L., and Gladwin, M.T. 2006. Ceruloplasmin is a NO oxidase and nitrite synthase that determines endocrine NO homeostasis. Nat Chem Biol 2: 486-493. doi: 10.1038/nchembio813.

Sokolov, A.V., Acquasaliente, L., Kostevich, V.A., Frasson, R., Zakharova, E.T., Pontarollo, G., Vasilyev, V.B., and De Filippis V. 2015a. Thrombin inhibits the anti-myeloperoxidase and ferroxidase functions of ceruloplasmin: relevance in rheumatoid arthritis. Free Radic Biol Med 86: 279-294. doi: 10.1016/j.freeradbiomed.2015.05.016.

Sokolov, A.V., Golenkina, E.A., Kostevich, V.A., Vasilyev, V.B., and Sud'ina, G.F. 2010. Interaction of ceruloplasmin and 5-lipoxygenase. Biochemistry (Mosc) 75: 1464-1469. doi: $10.1134 /$ S0006297910120072.

Sokolov, A.V., Kostevich, V.A., Zakharova, E.T., Samygina, V.R., Panasenko, O.M., and Vasilyev, V.B. 2015b. Interaction of ceruloplasmin with eosinophil peroxidase as compared to its interplay with myeloperoxidase: Reciprocal effect on enzymatic properties. Free Radic Res 49: 800-811. doi: 10.3109/10715762.2015.1005615.

Sokolov, A.V., Pulina, M.O., Ageeva, K.V., Runova, O.L., Zakharova, E.T., and Vasil'ev, V.B. 2007. Identification of leukocyte cationic proteins that interact with ceruloplasmin. Biochemistry (Mosc) 72: 872-877. doi: 10.1134/S0006297907080093.

Sokolov, A.V., Zakharova, E.T., Kostevich, V.A., Samygina, V.R., and Vasilyev, V.B. 2014. Lactoferrin, myeloperoxidase, and ceruloplasmin: complementary gearwheels cranking physiological and pathological processes. Biometals 27: 815-828. doi: 10.1007/s10534-014-9755-2.

Sud'ina, G.F., Brock, T.G., Pushkareva, M.A., Galkina, S.I., Turutin, D.V., Peters-Golden, M., and Ullrich, V. 2001. Sulphatides trigger polymorphonuclear granulocyte spreading on collagen-coated surfaces and inhibit subsequent activation of 5-lipoxygenase. Biochem J 359: 621-629. doi: 10.1042/bj3590621.

Takei, H., Araki, A., Watanabe, H., Ichinose, A., and Sendo, F. 1996. Rapid killing of human neutrophils by the potent activator phorbol 12-myristate 13-acetate (PMA) accompanied by changes different from typical apoptosis or necrosis. J Leukoc Biol 59: 229-240.

Tauber, A.I., Brettler, D.B., Kennington, E.A., and Blumberg, P.M. 1982. Relation of human neutrophil phorbol ester receptor occupancy and NADPH-oxidase activity. Blood 60: 333-339.

Varfolomeeva, E.Y., Semenova, E.V., Sokolov, A.V., Aplin, K.D., Timofeeva, K.E., Vasilyev, V.B., and Filatov, M.V. 2016. Ceruloplasmin decreases respiratory burst reaction during pregnancy. Free Radic Res 50: 909-919. doi: 10.1080/10715762.2016.1197395. 
Viryasova, G.M., Galkina, S.I., Gaponova, T.V., Romanova, J.M., and Sud'ina, G.F. 2014. Regulation of 5oxo-ETE synthesis by nitric oxide in human polymorphonuclear leucocytes upon their interaction with zymosan and Salmonella typhimurium. Biosci Rep 34: e00108. doi: 10.1042/BSR20130136.

Viryasova, G.M., Golenkina, E.A., Galkina, S.I., Gaponova, T.V., Romanova, Y.M., and Sud'ina, G.F. 2016. Effects of phosphodiester and phosphorothioate ODN2216 on leukotriene synthesis in human neutrophils and neutrophil apoptosis. Biochimie 125: 140-149. doi: 10.1016/j.biochi.2016.03.010.

Weitzel, F., and Wendel, A. 1993. Selenoenzymes regulate the activity of leukocyte 5-lipoxygenase via the peroxide tone. J Biol Chem 268: 6288-6292.

Williams, J.D., Lee, T.H., Lewis, R.A., and Austen, F. 1985. Intracellular retention of the 5-lipoxygenase pathway product, leukotriene $B 4$, by human neutrophils activated with unopsonized zymosan. J Immunol 134: 2624-2630.

Winterbourn, C.C., Garcia, R.C., and Segal, A.W. 1985. Production of the superoxide adduct of myeloperoxidase (compound III) by stimulated human neutrophils and its reactivity with hydrogen peroxide and chloride. Biochem J 228: 583-592. doi: 10.1042/bj2280583.

Zschaler, J., and Arnhold, J. 2016. Impact of simultaneous stimulation of 5-lipoxygenase and myeloperoxidase in human neutrophils. Prostaglandins Leukot Essent Fatty Acids 107: 12-21. doi: 10.1016/j.plefa.2016.02.001.

Zschaler, J., Dorow, J., Schöpe, L., Ceglarek, U., and Arnhold, J. 2015. Impact of myeloperoxidase-derived oxidants on the product profile of human 5-lipoxygenase. Free Radic Biol Med 85: 148-156. doi: 10.1016/j.freeradbiomed.2015.04.015 
318 Figure Legends

319 Fig. 1. Effects of P1 and P2 on the formation of 5-LOX products in human PMNLs.

320 PMNLs $\left(2 \times 10^{7}\right)$ were incubated for $30 \mathrm{~min}$ at $37^{\circ} \mathrm{C}$ without additives or with $20 \mu \mathrm{M} \mathrm{P} 1$ or P2

321 plus PMA $(0.5 \mathrm{nM})$, as indicated, and then added to a solution containing $0.5-1 \times 10^{9}$ Salmonella

322 Typhimurium bacterial cells for $20 \mathrm{~min}$. The products of arachidonic acid transformation by 5-

323 LOX were extracted from the medium and separated using HPLC. LTs; the sum of LTB4, its

324 isomers, and $\omega$-OH-LTB4. Values indicate means \pm SEMs for six independent experiments

325 performed in duplicate. ${ }^{*} P<0.05$ compared with the control. ${ }^{\sharp} P<0.05$ for pairs of data

326 compared as indicated by one-way ANOVA followed by Holm-Š́ídák multiple comparison tests.

327 Fig. 2. Effects of P1 and P2 on superoxide formation in PMNLs.

328

329

PMNLs $\left(1 \times 10^{6} / \mathrm{mL}\right)$ were seeded in 24 -well plates in HBSS-HEPES medium with $50 \mu \mathrm{M}$ cyto

330

c and $20 \mu \mathrm{M}$ P1 or P2, with or without $0.5 \mathrm{nM}$ PMA and $30 \mathrm{U} / \mathrm{mL}$ superoxide dismutase (SOD).

Cells were then incubated at $37^{\circ} \mathrm{C}$ for $40 \mathrm{~min}$. Cyto c reduction was measured by determining

changes in absorbance at 555 and $531 \mathrm{~nm}$. Values indicate the means \pm SDs from six

332

independent experiments performed in duplicate. ${ }^{\#} P<0.05$ for pairs of data compared as

333

indicated by one-way ANOVA followed by Holm-Š́́dák multiple comparison tests.

334

Fig. 3. Effects of P1 and P2 on ROS formation in PMNLs.

PMNLs were treated for $1 \mathrm{~h}$ with $5 \mu \mathrm{M}$ carboxy-H2DCFDA, washed, and suspended in

HBSS/HEPES medium. Cells $\left(1 \times 10^{6} / \mathrm{mL}\right)$ were seeded in 24 -well plates with $20 \mu \mathrm{M} \mathrm{P1}$ or P2,

with or without $0.5 \mathrm{nM}$ PMA, and incubated at $37^{\circ} \mathrm{C}$ for $60 \mathrm{~min}$. $\mathrm{ROS}$ formation was evaluated

by measurement of fluorescence. Values indicate the means \pm SDs from three independent

experiments performed in duplicate. ${ }^{* *} P<0.01$ compared with the control. ${ }^{\# \#} P<0.01$ for pairs

340

of data compared as indicated by one-way ANOVA followed by Holm-Š́ídák multiple

341 comparison tests.

342

Scheme 1. Scheme of P2 peptide activity on MPO-mediated ROS formation. 


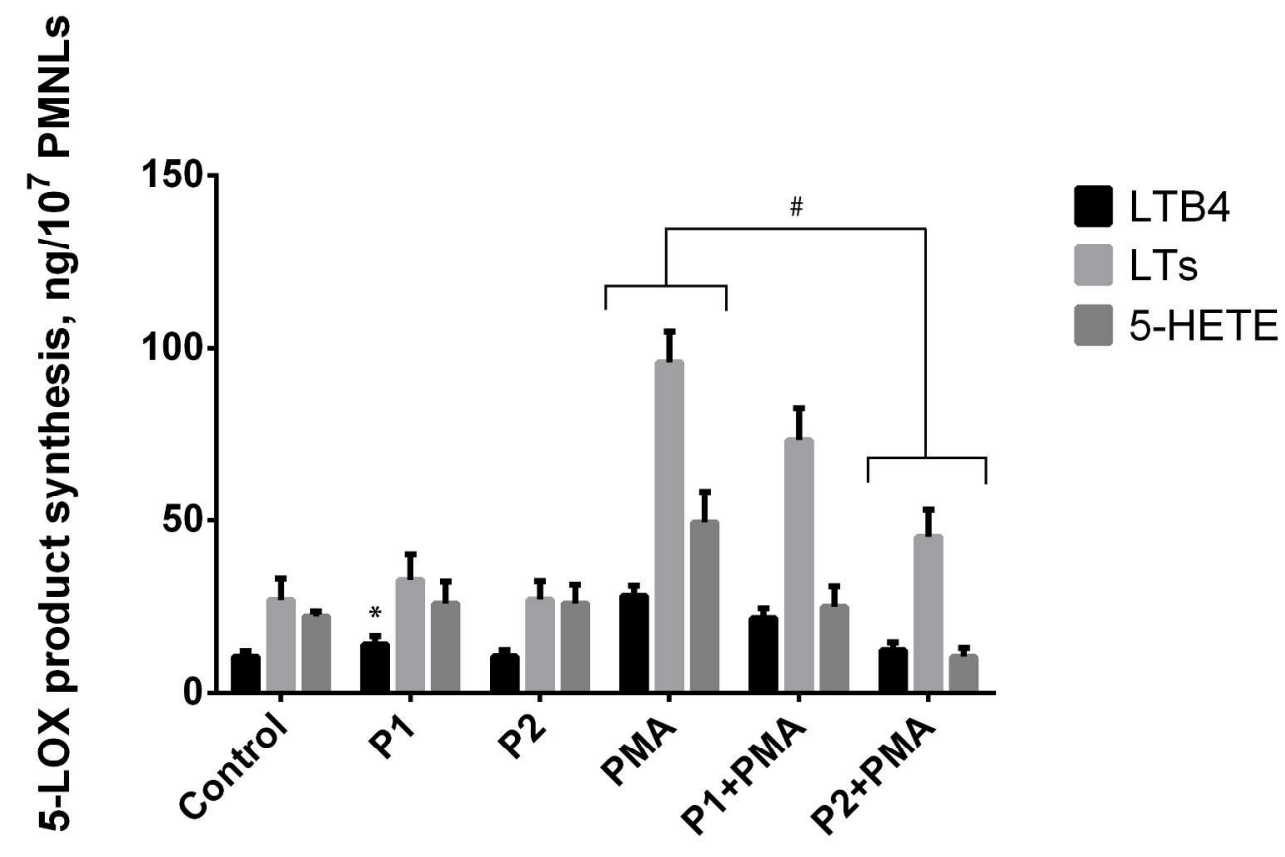

Fig. 1. Effects of $\mathrm{P} 1$ and $\mathrm{P} 2$ on the formation of 5-LOX products in human PMNLs.

PMNLs $(2 \times 107)$ were incubated for $30 \mathrm{~min}$ at $37^{\circ} \mathrm{C}$ without additives or with $20 \mu \mathrm{M}$ P1 or P2 plus PMA (0.5 $\mathrm{nM})$, as indicated, and then added to a solution containing 0.5-1 $\times 109$ Salmonella Typhimurium bacterial cells for $20 \mathrm{~min}$. The products of arachidonic acid transformation by 5-LOX were extracted from the medium and separated using HPLC. LTs; the sum of LTB4, its isomers, and $\omega$-OH-LTB4. Values indicate means \pm SEMs for six independent experiments performed in duplicate. $* P<0.05$ compared with the control. \#P $<$ 0.05 for pairs of data compared as indicated by one-way ANOVA followed by Holm-Šídák multiple comparison tests.

In the presence of PMA, P2 app

$215 \times 145 \mathrm{~mm}(300 \times 300 \mathrm{DPI})$ 


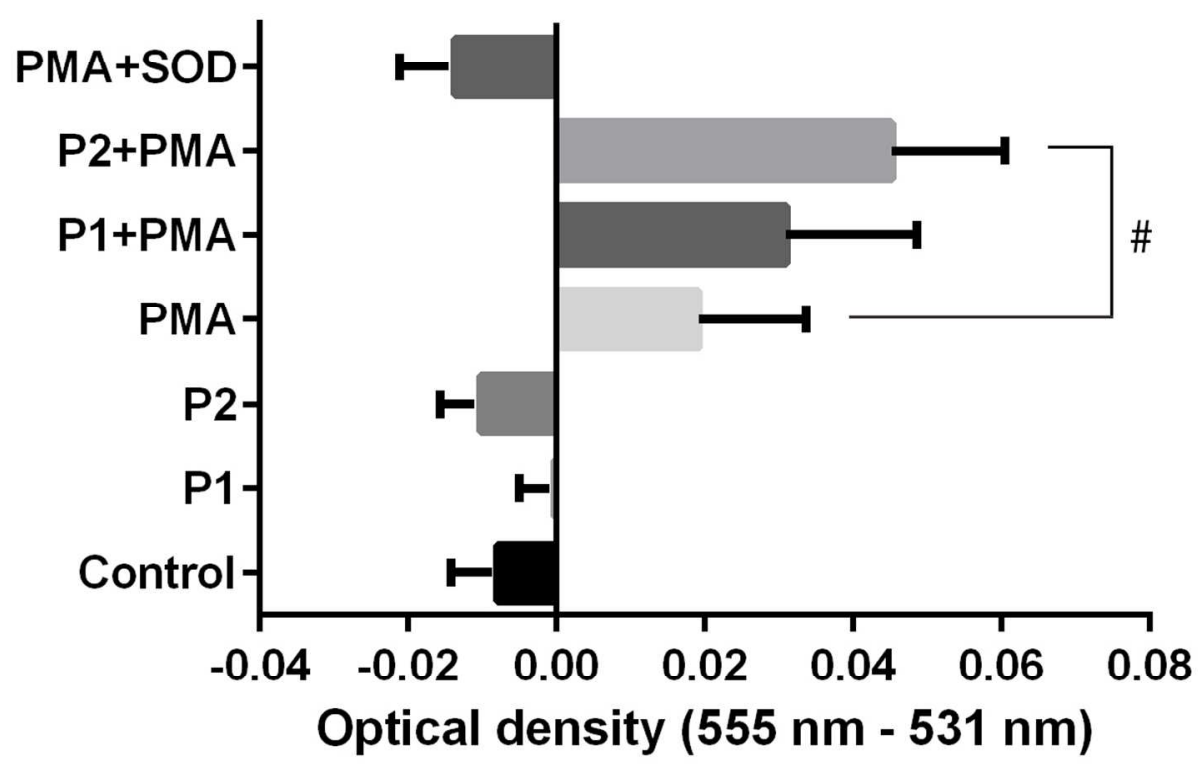

Fig. 2. Effects of $P 1$ and $P 2$ on superoxide formation in PMNLs.

PMNLs $(1 \times 106 / \mathrm{mL})$ were seeded in 24-well plates in HBSS-HEPES medium with $50 \mu \mathrm{M}$ cyto c and $20 \mu \mathrm{M}$ P1 or $\mathrm{P} 2$, with or without $0.5 \mathrm{nM} P M A$ and $30 \mathrm{U} / \mathrm{mL}$ superoxide dismutase (SOD). Cells were then incubated at $37^{\circ} \mathrm{C}$ for $40 \mathrm{~min}$. Cyto $\mathrm{C}$ reduction was measured by determining changes in absorbance at 555 and $531 \mathrm{~nm}$. Values indicate the means \pm SDs from six independent experiments performed in duplicate. \#P $<0.05$ for pairs of data compared as indicated by one-way ANOVA followed by Holm-Šídák multiple comparison tests. superoxide formation (Fig. 2).

$111 \times 73 \mathrm{~mm}$ (300 x $300 \mathrm{DPI})$ 


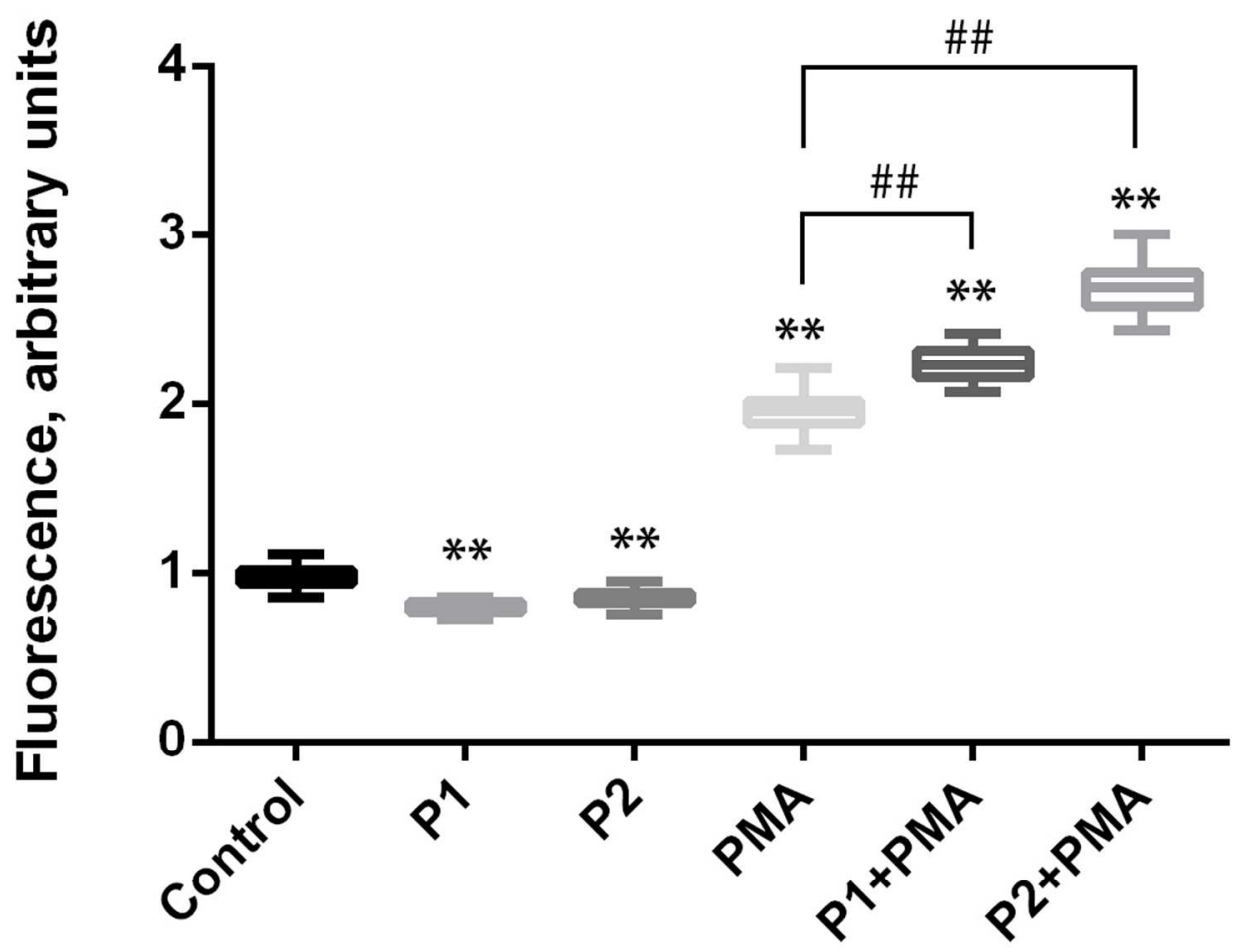

Fig. 3. Effects of $P 1$ and $P 2$ on ROS formation in PMNLs.

PMNLs were treated for $1 \mathrm{~h}$ with $5 \mu \mathrm{M}$ carboxy-H2DCFDA, washed, and suspended in HBSS/HEPES medium. Cells $(1 \times 106 / \mathrm{mL})$ were seeded in 24-well plates with $20 \mu \mathrm{M}$ P1 or P2, with or without $0.5 \mathrm{nM}$ PMA, and incubated at $37^{\circ} \mathrm{C}$ for $60 \mathrm{~min}$. ROS formation was evaluated by measurement of fluorescence. Values indicate the means \pm SDs from three independent experiments performed in duplicate. $* * P<0.01$ compared with the control. \#\#P $<0.01$ for pairs of data compared as indicated by one-way ANOVA followed by Holm-Šídák multiple comparison tests.

Without PMA, P1 and P2 decreas

$97 \times 77 \mathrm{~mm}(300 \times 300 \mathrm{DPI})$ 


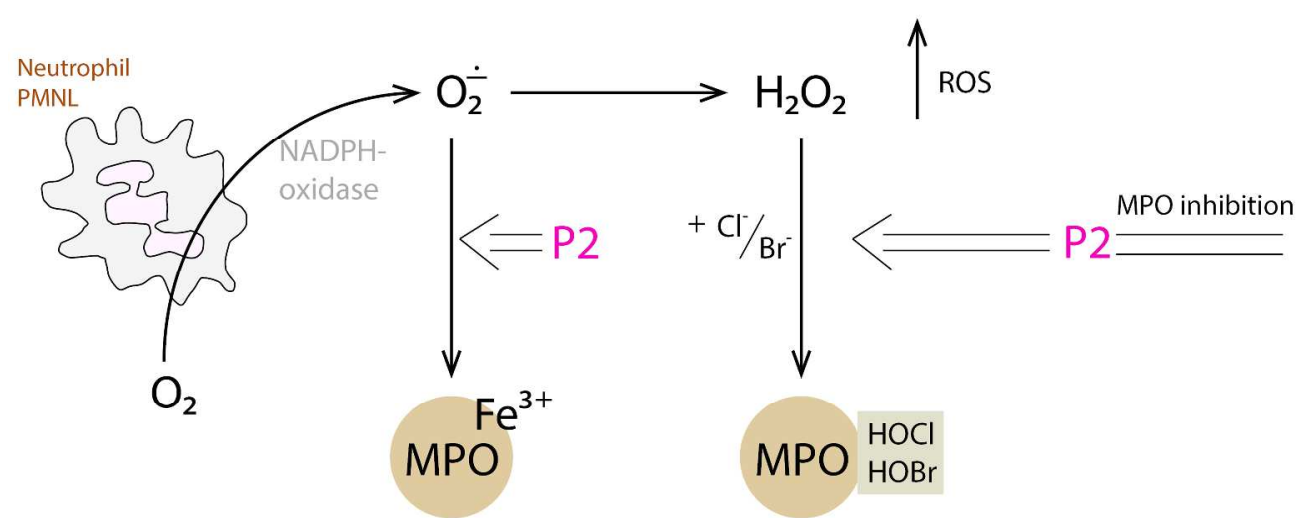

Scheme 1. Scheme of P2 peptide activity on MPO-mediated ROS formation. is blocked (Scheme 1). P2 pept

$291 \times 117 \mathrm{~mm}$ (300 x 300 DPI) 